\title{
Fibrinolysis with Lower Dose Urokinase in Patients with Complicated Parapneumonic Effusion
}

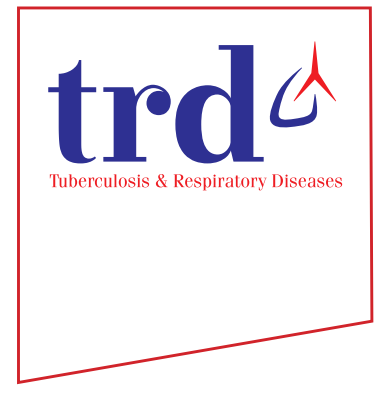

\author{
Seul Lee, M.D. ${ }^{1 \oplus}$, Heock Lee, M.D. ${ }^{1}$, Dong Hyun Lee, M.D. ${ }^{1,2}$, Bo Hyoung Kang, M.D. ${ }^{1}$, Mee Sook \\ Roh, M.D. ${ }^{3}$, Choohee Son, M.D. ${ }^{1}$, Sung Hyun Kim, M.D. ${ }^{4}$, Hyun-Kyung Lee, M.D. ${ }^{4}$ and Soo-Jung \\ Um, M.D. ${ }^{1}$ (i)
}

${ }^{1}$ Pulmonology Division, Department of Internal Medicine, ${ }^{2}$ Department of Intensive Care Medicine, ${ }^{3}$ Department of Pathology, Dong-A University Hospital, Dong-A University College of Medicine, Busan, ${ }^{4}$ Division of Pulmonology, Allergy and Critical Care Medicine, Department of Internal Medicine, Busan Paik Hospital, Inje University College of Medicine, Busan, Republic of Korea

Background: Intrapleural urokinase is one of the most widely used fibrinolytic agents in the treatment of complicated parapneumonic effusion (CPPE). However, little research has been performed on the optimal urokinase dosage. The aim of this study was to evaluate the treatment efficacy of half dose urokinase compared with conventional dose urokinase.

Methods: We retrospectively enrolled 92 patients with CPPE or empyema who underwent intrapleural urokinase treatment at two tertiary hospitals. Patients received antibiotics, chest tube drainage, and other treatments as part of routine care. The primary outcome was the treatment success rate in the half dose urokinase group (50,000 IU daily for maximal 6 days) and the conventional dose urokinase group (100,000 IU daily). Treatment success was defined as clinical and radiological improvements without surgical treatment or re-admission within one month.

Results: Forty-four patients received half dose urokinase, whereas 48 patients were treated with conventional dose urokinase. Both groups were relatively well matched at baseline, excluding higher serum white blood cell count and higher empyema prevalence in the half dose urokinase group. The treatment success rate was not different between the two groups ( $\mathrm{p}=0.048$ ). There were no differences in the rate of in-hospital death and surgical treatment, hospitalization duration, and indwelling catheter duration. In the multivariate analysis, urokinase dose was not a predictor of treatment success. Conclusion: Half dose intrapleural urokinase is equally effective conventional dose urokinase in treating patients with CPPE or empyema.

Keywords: Catheters; Empyema; Fibrinolysis; Indwelling; Pleurisy; Pneumonia

Address for correspondence: Soo-Jung Um, M.D.

Pulmonology Division, Department of Internal Medicine, Dong-A University Hospital, Dong-A University College of Medicine, 26 Daesingongwon-ro, Seo-gu, Busan 49201, Republic of Korea Phone: 82-51-240-2769, Fax: 82-51-242-5852

E-mail: sjum@dau.ac.kr

Received: Aug. 7, 2020

Revised: Oct. 22, 2020

Accepted: Dec. 15, 2020

Published online: Dec. 17, 2020

(c) It is identical to the Creative Commons Attribution Non-Commercial License (http://creativecommons.org/licenses/by-nc/4.0/). The Korean Academy of Tuberculosis and Respiratory Diseases.

\section{Introduction}

Parapneumonic effusion is the filling of the pleural cavity with exudative pleural fluid, which develops secondary to pneumonia. Although the majority of parapneumonic effusion is improved with antibiotic treatment, some of this condition progress from uncomplicated parapneumonic effusion to complicated parapneumonic effusion (CPPE) or even to empyema. The basic principle of treating CPPE includes an appropriate antibiotic treatment and adequate drainage of the infected fluid collection ${ }^{1}$. Percutaneous catheter drainage is the main interventional modality to decrease the amount of CPPE; however, this approach is not always successful owing to a thick layer of fibrin deposit and loculated nature of effu- 
sions. Therefore, intrapleural fibrinolytic treatment has been widely employed to lyse the fibrinous structures in pleural space $^{2-5}$.

Urokinase is one of the most widely used fibrinolytic agents ${ }^{3}$; however, its proper dosage remains entirely empiric. Because most previous studies used more than 100,000 IU for single fibrinolysis, conventional single dose has been thought to be $100,000 \mathrm{IU}^{6}$. Fibrinolysis inherently accompanies the risk of hemorrhagic complications and is relatively expensive treatment; therefore, the concept of lower dose urokinase was raised by some physicians. The authors have taken lower dose strategy for several years to reduce side effects and enhance cost-effectiveness. However, there has been no evidence of low dose fibrinolytic treatment. We herein sought to investigate the treatment efficacy of half dose urokinase compared with conventional dose urokinase.

\section{Materials and Methods}

\section{Patients}

We retrospectively reviewed medical records of patients with CPPE or empyema who had received urokinase fibrinolysis from January 2013 to December 2016 at the Dong-A University Hospital and Inje University Busan Paik Hospital. CPPE referred to those pneumonia associated with effusion demonstrated by imaging studies such as chest X-ray, computed tomography (CT) or ultrasound and one of the following pleural fluid characteristics: $\mathrm{pH}<7.20$, glucose $<60 \mathrm{mg} / \mathrm{dL}$, lactate dehydrogenase $>3$ times of normal limit, or positive Gram stain or culture ${ }^{7}$. Empyema was defined as gross pus within the pleural space. We also reviewed radiologic manifestations regarding the severity of CPPE or empyema, which were bilateral involvement, the amount of effusion above $1 / 3$ of hemithorax, the presence of air-fluid level on chest CT, the presence of multiple loculation, and the thickness of effusion above $3 \mathrm{~cm}$ on chest CT. Patients with non-diagnostic criteria for CPPE such as transudate effusion, tuberculous pleurisy or malignant pleural effusion were excluded. Other exclusion criteria were as follows: pregnancy or breast-feeding period, terminal clinical condition, coagulopathy, anti-coagulant therapy, dual anti-platelet therapy, broncho-pleural fistula, active bleeding in any location, and the previous use of urokinase. This study was approved by the Dong-A University Hospital and In-Je University Hospital Institutional Review Board (DAUHIRB-20-082, 20-0062) and conducted in accordance with the ethical guidelines of the Declaration of Helsinki. The written or oral informed consent was waived because of the retrospective study design.

\section{Treatment}

Urokinase dose as well as empirical antibiotic treatment were left at the discretion of the attending physicians. The following intrapleural fibrinolysis regimens were instituted until a recognition of treatment success. Half dose group: urokinase $50,000 \mathrm{IU}$ in $20 \mathrm{~mL}$ saline every 24 hours, conventional dose group: urokinase 100,000 IU in $20 \mathrm{~mL}$ saline every 24 hours. Half dose regimen was predominantly used in the Dong-A University Hospital, whereas conventional dose regimen was mainly prescribed in the Inje university hospital. A small-bore chest tube was inserted into the lowest area of the pleural effusion or, in the case of multiloculated effusions, into the largest loculation. After the first instillation of fibrinolytics, the catheter key was closed for two hours and then reopened. Additional daily urokinase doses were prescribed if pleural fluid collections remained visible on imaging studies for maximal 6 days. Surgical debridement was performed when there was persistence of clinical signs and symptoms of pleural infection in association with a substantial residual pleural collection.

\section{Evaluation outcomes}

The primary outcome was treatment success. The treatment success was defined as the improvement of clinical symptoms and signs of pleural infection including fever, pleuritic chest pain, and serum inflammatory markers along with resolution or considerable abatement of chest radiologic findings without surgical treatment, re-admission within 1 month after index hospital discharge. Fibrinolytic treatment was deemed to be failed in the absence of all fulfillment of success criteria. The secondary outcomes included in-hospital death, surgical treatment, hospitalization duration, the number of fibrinolysis, total drained amount of pleural effusion, and indwelling catheter duration.

\section{Statistical analysis}

Continuous data are presented as means and standard deviation. Differences in continuous variables were determined using the t-test or the Mann-Whitney U test. Categorical data are presented as frequencies. Differences between categorical variables were analyzed using the Pearson's chi-square test or the Fisher exact test. We used multivariate logistic regression analysis to estimate the independent factors for treatment success with fibrinolysis adjusting the models for the age, sex, pneumonia severity index score, severe involvement based on radiologic finding, and urokinase dose. Severe radiologic involvement was defined as the presence of at least three of the following radiologic findings: bilateral involvement, the amount of effusion above $1 / 3$ of hemithorax, the presence of air-fluid level on chest CT, the presence of multiple loculation, and the thickness of effusion above $3 \mathrm{~cm}$ on chest CT. A p-val- 
ue $<0.05$ was considered statistically significant. All statistical analyses were performed using SPSS version 20.0 (IBM Corp., Armonk, NY, USA).

\section{Results}

Among 1,250 patients diagnosed as having pneumonia, 92 patients with CPPE or empyema who need intrapleural urokinase treatment were enrolled in this study, of whom 44 were treated with daily half dose urokinase 50,000 IU and 48 received daily conventional dose urokinase 100,000 IU. Both groups were homogeneous with regard to clinical and pleural fluid characteristics (Tables 1, 2), although pre/post-urokinase serum white blood cell count and the prevalence of empyema were higher in the half dose group. The radiologic findings suggestive of severe involvement did not show statistical differences in the both groups (Table 3 ).

Table 4 shows the clinical outcomes of patients treated with intrapleural urokinase. The treatment success rate was not different between two groups ( $\mathrm{p}=0.048$ ). There were eight failures $(18.2 \%)$ in the half dose group and eight failures (16.7\%) in the conventional dose group. Each one patient in the both group died during hospitalization. Each two patients received surgical treatment owing to ineffective intrapleural urokinase treatment. Six patients in the half dose group and five patients in the conventional dose group were transferred to local convalescent hospitals without clinical improvement. As shown in Table 5, a significantly smaller amount of urokinase was administered in the half dose group, although the number of

Table 1. Demographic and comorbidity data of the study population

\begin{tabular}{|lcccc|}
\hline & $\begin{array}{c}\text { Overall } \\
(\mathbf{n = 9 2 )}\end{array}$ & $\begin{array}{c}\text { Half dose } \\
\mathbf{5 0 , 0 0 0 ~ I U ~ ( n = 4 4 )}\end{array}$ & $\begin{array}{c}\text { Conventional dose } \\
\mathbf{1 0 0 , 0 0 0 ~ I U ~ ( n = 4 8 )}\end{array}$ & p-value \\
\hline Age, yr & $62.1 \pm 14.2$ & $64.9 \pm 13.4$ & $59.5 \pm 14.6$ & 0.071 \\
\hline Male sex & $73(79.3)$ & $36(81.8)$ & $37(77.1)$ & 0.615 \\
\hline PSI & $98.1 \pm 26.5$ & $96.1 \pm 28.2$ & $99.9 \pm 25.0$ & 0.494 \\
\hline Empyema & $16(17.4)$ & $13(29.5)$ & $3(6.3)$ & 0.005 \\
\hline Diabetes & $21(22.8)$ & $14(31.8)$ & $7(14.6)$ & 0.080 \\
\hline CV disease & $29(31.5)$ & $17(38.6)$ & $12(25.0)$ & 0.183 \\
\hline CNS disease & $22(23.9)$ & $13(29.5)$ & $9(18.8)$ & 0.328 \\
\hline Liver disease & $4(4.3)$ & $3(6.8)$ & $1(2.1)$ & 0.346 \\
\hline Renal disease & $2(2.2)$ & $1(2.3)$ & $1(2.1)$ & $>0.99$ \\
\hline Malignancies & $7(7.6)$ & $5(11.4)$ & $2(4.2)$ & 0.253 \\
\hline
\end{tabular}

Values are presented as mean \pm standard deviation or number $(\%)$.

PSI: pneumonia severity index; CV: cardiovascular; CNS: central nervous system.

Table 2. Blood and pleural fluid characteristics

\begin{tabular}{|c|c|c|c|}
\hline & $\begin{array}{c}\text { Half dose } \\
50,000 \text { IU }(n=44)\end{array}$ & $\begin{array}{l}\text { Conventional dose } \\
100,000 \text { IU }(n=48)\end{array}$ & p-value \\
\hline \multicolumn{4}{|l|}{ Blood } \\
\hline Pre-UK WBC, $\times 10^{3} / \mu \mathrm{L}$ & $18.0 \pm 8.1$ & $14.2 \pm 6.9$ & 0.018 \\
\hline Post-UK WBC, $\times 10^{3} / \mu \mathrm{L}$ & $10.5 \pm 4.7$ & $82 \pm 49$ & 0.025 \\
\hline Pre-UK CRP, mg/dL & $23.0 \pm 9.1$ & $19.4 \pm 10.4$ & 0.081 \\
\hline Post-UK CRP, mg/dL & $6.3 \pm 5.6$ & $4.2 \pm 5.7$ & 0.078 \\
\hline \multicolumn{4}{|l|}{ Pleural fluid } \\
\hline $\mathrm{pH}$ & $6.9 \pm 0.3$ & $6.8 \pm 0.3$ & 0.895 \\
\hline Glucose, mg/dL & $56.9 \pm 73.4$ & $70.0 \pm 72.4$ & 0.389 \\
\hline Lactate dehydrogenase, $\mathrm{U} / \mathrm{L}$ & $3,981.3 \pm 4,399.3$ & $2,733.3 \pm 5,565.3$ & 0.239 \\
\hline Neutrophil, $\times 10^{3} / \mu \mathrm{L}$ & $28.5 \pm 96.4$ & $10.9 \pm 14.1$ & 0.214 \\
\hline
\end{tabular}

Values are presented as mean \pm standard deviation.

UK: urokinase; WBC: white blood cell; CRP: C-reactive protein. 
Table 3. The severity of radiologic manifestations according to the urokinase dose

\begin{tabular}{|lccc|}
\hline & $\begin{array}{c}\text { Half dose } \\
\mathbf{5 0 , 0 0 0} \text { IU (n=44) }\end{array}$ & $\begin{array}{c}\text { Conventional dose } \\
\mathbf{1 0 0 , 0 0 0} \text { IU (n=48) }\end{array}$ & p-value \\
\hline Bilateral involvement & $6(13.6)$ & $3(6.3)$ & 0.302 \\
\hline Effusion amount $\geq 1 / 3$ of the hemithorax & $39(88.6)$ & $39(81.3)$ & 0.392 \\
\hline Air-fluid level* & $9(20.5)$ & $8(16.7)$ & 0.640 \\
\hline Multiple loculations & $26(59.1)$ & $21(43.8)$ & 0.141 \\
\hline Effusion thickness $\geq 3$ cm* $^{*}$ & $41(93.2)$ & $45(93.8)$ & $>0.99$ \\
\hline
\end{tabular}

Values are presented as number (\%).

*Findings based on chest computed tomography.

Table 4. Clinical outcomes according to the urokinase dose

\begin{tabular}{|lccc|}
\hline & $\begin{array}{c}\text { Half dose } \\
\mathbf{5 0 , 0 0 0} \text { IU }(\mathbf{n = 4 4 )}\end{array}$ & $\begin{array}{c}\text { Conventional dose } \\
\mathbf{1 0 0 , 0 0 0} \text { IU (n=48) }\end{array}$ & p-value \\
\hline Treatment success & $36(81.8)$ & $40(83.3)$ & 0.848 \\
In-hospital death & $1(2.3)$ & $1(2.1)$ & $>0.99$ \\
\hline Surgical treatment & $2(4.5)$ & $2(4.2)$ & $>0.99$ \\
Hospitalization duration, day & $21.9 \pm 23.1$ & $19.5 \pm 8.1$ & 0.504 \\
\hline Referral to a local hospital & $6(13.6)$ & $5(10.4)$ & 0.752 \\
\hline
\end{tabular}

Values are presented as number (\%) or mean \pm standard deviation.

Table 5. Procedural outcomes according to the urokinase dose

\begin{tabular}{|lccc|}
\hline & $\begin{array}{c}\text { Half dose } \\
\mathbf{5 0 , 0 0 0} \mathbf{~ I U}(\mathbf{n}=\mathbf{4 4})\end{array}$ & $\begin{array}{c}\text { Conventional dose } \\
\mathbf{1 0 0 , 0 0 0} \text { IU (n=48) }\end{array}$ & p-value \\
\hline Total urokinase amount, $\times 10^{4} \mathrm{IU}$ & $18.5 \pm 14.2$ & $37.1 \pm 27.4$ & $<0.001$ \\
\hline No. of fibrinolysis & $3.7 \pm 2.8$ & $3.7 \pm 2.7$ & 0.964 \\
\hline Total amount of drained effusion, $\mathrm{mL}$ & $1,332.0 \pm 1,237.4$ & $1,467.6 \pm 1,003.5$ & 0.566 \\
\hline Indwelling catheter duration, day & $10.4 \pm 6.3$ & $11.9 \pm 6.2$ & 0.235 \\
\hline
\end{tabular}

Values are presented as mean \pm standard deviation.

fibrinolysis, total amount of drained effusion through percutaneous catheter, and indwelling catheter duration was not different between the two groups. There was no clinically relevant bleeding event in both groups. In the multivariate logistic regression analysis, urokinase dose was not a factor to predict treatment success or failure. Only severe radiologic involvement was a significant predictor for treatment failure (odds ratio, 6.204; 95\% confidence interval, $1.247-30.871 ; \mathrm{p}=0.026$ ).

\section{Discussion}

In this retrospective observational series enrolling patients from real-world clinical practice, half dose urokinase 50,000 IU is equally as effective as conventional dose $100,000 \mathrm{IU}$ to treat patient with CPPE with or without empyema who need percutaneous drainage. The results of this study demonstrat- ed the following. First, there were no significant differences in the rate of treatment success between two groups. Second, the rate of in-hospital death or surgical treatment were also not significantly different. Third, intrapleural urokinase fibrinolysis was a useful therapeutic approach for the majority of patients irrespective of the dose.

Since the successful fibrinolysis using streptokinase ${ }^{8}$, there have been numerous reports of fibrinolysis to treat patients with CPPE. Fibrinolysis using urokinase was first reported by Moulton et al. . In their study, the empirical urokinase doses of 80,000-150,000 IU were used without specific explanation for choosing such doses. In the following studies, urokinase dose varied from 100,000 IU to 250,000 IU $\mathrm{IU}^{10-13}$. Recently, streptokinase and urokinase are the most widely used fibrinolytic agents and guideline recommend 250,000 IU for streptokinase and 100,000 IU for urokinase as usual dose ${ }^{6}$. However, recommended urokinase dose is mainly based on 
small observational studies, and dose is frequently to change in real clinical practice. Bouros et al. ${ }^{14}$ firstly reported a study regarding the clinical applicability of fibrinolysis using lower dose urokinase, in which patients with CPPE and empyema were successfully treated with urokinase 50,000 IU. However, the number of enrolled patients was only 20 and this half dose was not compared with conventional higher dose 100,000 IU. To the best of our knowledge, our study is the first study to compare the fibrinolysis outcomes between half dose urokinase and conventional dose. Our study showed high treatment success rate and low surgical treatment rate. The need for surgical treatment has been an important endpoint in previous studies regarding intrapleural fibrinolysis and recent meta-analysis concluded that fibrinolysis alone may prevent the need of surgical drainage ${ }^{15}$. What is notable in our study is that a minority of patients in the half dose group (4.5\%) as well as in the conventional dose group (4.2\%) underwent surgical treatment. These values are in contrast to the $16 \%$ of patients requiring surgical referral within 3 months of having received streptokinase in the MIST1 study (first multicenter intrapleural sepsis trial ${ }^{16}$. Recent advances in treatment other than intrapleural fibrinolysis for CPPE patients may contribute to these low surgical treatment rates. Another notable point in our study is that the total amount of drained effusion during catheter indwelling time was not statistically different between the both groups. This result supports that the lower dosage of the UK shows the same clinical and radiologic recovery under the similar severity of CPPE.

Intrapleural fibrinolysis with lower dose urokinase is reasonable in several aspects. Urokinase is an expensive drug, therefore, the lower urokinase dose would result in a reduction of medical expense. In addition, although the incidence is low, intrapleural fibrinolysis accompanies the possibility of bleeding complications ${ }^{17,18}$, so lower urokinase dose might decrease bleeding events in vulnerable patients. Since increased dose and interval of fibrinolysis was associated with increased treatment efficacy in previous experimental study ${ }^{19}$, further prospective randomized clinical trial is warranted to reveal unknown benefit and pitfall of fibrinolysis using half dose urokinase.

This study has a few limitations. First, our study was a retrospective observational study. Therefore, the possibility of selection bias and/or residual confounding from unknown or unmeasured covariates cannot be excluded. However, we included relatively a large number of patients from two tertiary hospitals. Second, urokinase dose was unequally distributed by the participating hospitals. However, participating two hospitals had similar treatment policy except for the urokinase dose. In addition, both groups were relatively well-matched with regard to baseline clinical and laboratory characteristics. Even though serum white blood cell count and the prevalence of empyema, which might be poor prognostic factors, were higher in the half dose group, treatment success rate was not different between both groups. Furthermore, urokinase dose was not a predictor of treatment success in the multivariate analysis. Third, there is a possibility that our study included relatively milder patients compared to the MIST. Therefore, our results may not be applicable to patients with severe CPPE or empyema who need prior surgical treatment without fibrinolysis. Fourth, there were several patients whose final clinical outcomes were not confirmed because these patients were transferred to local hospitals without clinical improvement. Finally, long-term clinical outcomes were not evaluated in this study.

In conclusion, intrapleural fibrinolysis with half dose urokinase is equally as effective as conventional dose to treat patients with CPPE with or without empyema at possible lower cost.

\section{Authors' Contributions}

Conceptualization: Um SJ. Methodology: Um SJ, Lee HK, Son C. Formal analysis: Um SJ. Data curation: Lee S, Lee H, Kang BH, Kim SH, Lee HK. Writing - original draft preparation: Lee S, Lee DH. Writing - review \& editing: Um SJ, Roh MS, Son C, Lee HK. Approval of final manuscript: all authors.

\section{Conflicts of Interest}

No potential conflict of interest relevant to this article was reported.

\section{Funding}

This study was supported by research funds from Dong-A University.

\section{References}

1. Light RW. Parapneumonic effusions and empyema. Clin Chest Med 1985;6:55-62.

2. Kim YJ, Cha SI, Kwon JS, Yoo SS, Jun HJ, Kim EJ, et al. Treatment results and prognostic factors of complicated parapneumonic effusion and empyema. Tuberc Respir Dis 2007;63:2430 .

3. Park JW, You SM, Seol WJ, Paik EK, Lee KH, Seo JB, et al. Efficacy of pigtail catheter drainage in patients with thoracic empyema or complicated parapneumonic effusion. Tuberc Respir Dis 2003;54:219-29.

4. Kim YS, Kim SM, Kim JH, Lee KS, Yang SC, Yoon HJ, et al. The effect of percutaneous pig-tail catheter drainage in the management of lung abscess and empyema. Tuberc Respir Dis 
1996;43:571-8.

5. Janda S, Swiston J. Intrapleural fibrinolytic therapy for treatment of adult parapneumonic effusions and empyemas: a systematic review and meta-analysis. Chest 2012;142:401-11.

6. Davies HE, Davies RJ, Davies CW; BTS Pleural Disease Guideline Group. Management of pleural infection in adults: British Thoracic Society Pleural Disease Guideline 2010. Thorax 2010;65 Suppl 2:ii41-53.

7. Light RW, MacGregor MI, Ball WC Jr, Luchsinger PC. Diagnostic significance of pleural fluid $\mathrm{pH}$ and $\mathrm{PCO}_{2}$. Chest 1973;64:591-6.

8. Bergh NP, Ekroth R, Larsson S, Nagy P. Intrapleural streptokinase in the treatment of haemothorax and empyema. Scand J Thorac Cardiovasc Surg 1977;11:265-8.

9. Moulton JS, Moore PT, Mencini RA. Treatment of loculated pleural effusions with transcatheter intracavitary urokinase. AJR Am J Roentgenol 1989;153:941-5.

10. Lee KS, Im JG, Kim YH, Hwang SH, Bae WK, Lee BH. Treatment of thoracic multiloculated empyemas with intracavitary urokinase: a prospective study. Radiology 1991;179:771-5.

11. Couser JI Jr, Berley J, Timm EG. Intrapleural urokinase for loculated effusion. Chest 1992;101:1467-9.

12. Cohen ML, Finch IJ. Transcatheter intrapleural urokinase for loculated pleural effusion. Chest 1994;105:1874-6.

13. Pollak JS, Passik CS. Intrapleural urokinase in the treatment of loculated pleural effusions. Chest 1994;105:868-73.

14. Bouros D, Schiza S, Tzanakis N, Drositis J, Siafakas N. Intrapleural urokinase in the treatment of complicated parapneumonic pleural effusions and empyema. Eur Respir J 1996;9: 1656-9.

15. Nie W, Liu Y, Ye J, Shi L, Shao F, Ying K, et al. Efficacy of intrapleural instillation of fibrinolytics for treating pleural empyema and parapneumonic effusion: a meta-analysis of randomized control trials. Clin Respir J 2014;8:281-91.

16. Maskell NA, Davies CW, Nunn AJ, Hedley EL, Gleeson FV, Miller R, et al. U.K. controlled trial of intrapleural streptokinase for pleural infection. N Engl J Med 2005;352:865-74.

17. Goralski JL, Bromberg PA, Haithcock B. Intrapleural hemorrhage after administration of tPA: a case report and review of the literature. Ther Adv Respir Dis 2009;3:295-300.

18. Hendaus MA, Abushahin A. Intrapleural hemorrhage due to alteplase use in a 6 -year-old boy with pleural effusion. Int J Gen Med 2013;6:233-6.

19. Strange C, Allen ML, Harley R, Lazarchick J, Sahn SA. Intrapleural streptokinase in experimental empyema. Am Rev Respir Dis 1993;147:962-6. 